\title{
Parenting interventions for child injury, and interventions for the construction industry: systematic reviews
}

Two important new systematic reviews in quite different fields of injury prevention have recently been published in issue 4, 2007 of The Cochrane Library (http:// www.thecochranelibrary.com) released in October 2007.

The first review, by Kendrick and colleagues, ${ }^{1}$ examined parenting interventions for the prevention of unintentional injuries in children. Parenting interventions are designed to provide education and training in parenting skills, which help in the development of stronger relationships with children and management of difficult behavior. The authors highlight that such interventions have been shown in systematic reviews to be effective in a number of different settings and population groups. ${ }^{1}$ The current review aimed to examine whether the effect of such interventions extends to the prevention of unintentional injury in childhood and the promotion of child safety.

The review looks at 15 studies, including 11 randomized controlled trials (RCT). The interventions comprised multi-faceted parenting interventions and other support services; 11 were home visiting services and two were administered in pediatric practices. Most of the interventions were administered in families at risk of adverse health outcomes. The primary meta-analysis included nine RCTs and found that intervention families had a significantly lower risk of injury (RR 0.82, 95\% CI 0.71 to 0.95$)$. The authors concluded that parenting interventions delivered at home to families at risk of adverse health outcomes were likely to be effective in reducing unintentional injury. ${ }^{1}$
However, there is further work to be done in this area. Most of the interventions included in the review were complex, and the mechanisms underlying the reduction in injury remain unclear. The authors recommended that future trials explore this more comprehensively. Moreover, the trials included in the review were all conducted in high-income countries, and it is therefore unclear whether the results can be generalized to other settings. Nonetheless, this is an important review which suggests that provision of home visiting programs to "at risk" families may have substantial benefits in terms of prevention of child injury as well as maternal and child health.

The second new injury prevention review published by the Cochrane Injuries Group examined interventions for preventing injuries in the construction industry. ${ }^{2}$ Although occupational injury rates among construction workers are high and various injury control strategies have been introduced to address these, it is unclear how effective such strategies are in terms of preventing injury. Given the significant financial implications of construction injuries where workers compensation costs are substantial, an evidencebased occupational health and safety policy is vital.

The review authors included five interrupted time series in the review, and commented that overall methodological quality was low. The data from the included time series studies were extracted and re-analysed by the authors to obtain comparable and reliable effect sizes.
Interventions assessed included regulations, a safety campaign, and a drug-free workplace. The regulatory interventions included a vertical fall arrest standard on the risk of fatal falls in construction workers, a vertical fall arrest standard on the risk of non-fatal injuries in carpenters, and implementation of a regulatory trench and excavation standard with a targeted inspection program. Although the regulatory interventions showed no effect, possibly because of lack of implementation of regulations, both the safety campaign and the drug-free workplace showed both initial and sustained effects.

The authors concluded that there is limited evidence of effectiveness for safety campaigns and drug-free workplace, but commented that the quality of studies was poor. Both studies that showed an effect incorporated multiple and ongoing interventions that targeted the whole work organization, and combined educational and facilitative strategies with enforcement. However, the authors commented that there may be a need to maximize compliance of employers and workers with the prescribed safety measures. Further research in this field is urgently required to ensure good management of occupational injuries, in both high-income and low-income country settings.

For further information about the Cochrane Injuries Group or to find out how you can join our team of review authors, please see our website at http:// www.cochrane-injuries.lshtm.ac.uk/.

\section{R Ivers}

Correspondence to: Rebecca 0 Ivers, rivers@george. org.au

\section{REFERENCES}

1. Kendrick D, Barlow J, Hampshire A, et al. Parenting interventions for the prevention of unintentional injuries in childhood. Cochrane Database Syst Rev 2007; (4):CD006020.

2. van der Molen HF, Lehtola MM, Lappalainen J, et al. Interventions for preventing injuries in the construction industry. Cochrane Database Syst Rev 2007;(4):CD006251. 